\title{
Phenylpropanoid sex pheromone component in hemolymph of male Carambola fruit fly, Bactrocera carambolae (Diptera: Tephritidae)
}

\begin{abstract}
Males of the Carambola fruit fly, Bactrocera carambolae Drew \& Hancock (Diptera: Tephritidae) are strongly attracted to, and feed on methyl eugenol (ME) that exists as a plant secondary compound in over 480 plant species worldwide. Upon feeding on this highly potent attractant, the males convert ME into a phenylpropanoid, (E)-coniferyl alcohol (ECF), that is stored in the rectal gland prior to its release as a sex pheromone component during calling and courtship. Here, using a series of chemical and behavioural assays, we provide evidence for the presence of ECF in the hemolymph and suggest the latter's involvement in transport of ECF to the male rectal gland following consumption of ME. The greatest concentration of ECF was detected in the hemolymph at $3 \mathrm{~h}$ after feeding on ME and subsequently decreased, whereas accumulation of ECF in the rectal gland reached a maximum at 2 days post-feeding. Using male flies as biodetectors, fractions of $1.5-9.2 \mathrm{kDa}$ from fractionated hemolymph of ME-fed males were found to be attractive and contained ECF as sex pheromone. In addition, the significant increase in the total concentration of protein in hemolymph from ME-fed males compared with that of ME-deprived males suggests a direct protein carrier involvement in hemolymph transport of the sex pheromone in B. carambolae. All these results are further discussed in comparison with previous results obtained from its sibling species, the Oriental fruit fly-B. dorsalis.
\end{abstract}

Keyword: Bactrocera carambolae; Methyl eugenol; (E)-coniferyl alcohol; Hemolymph; Rectal gland; Sex pheromone 